\title{
Percepção dos enfermeiros sobre a visita pré-operatória de enfermagem
}

\author{
Nurses' perception nursing preoperative visit \\ Percepción sobre la visita preoperatoria de enfermería de las enfermeras
}

Marcelo Alexandre Albino Filho ${ }^{1 *}$, Regiane Franchini Batista², Edvaldo Aparecido da Cruz².

\section{RESUMO}

Objetivo: analisar a percepção dos enfermeiros sobre a operacionalização da visita pré-operatória e identificar o entendimento e dificuldades para realizá-la. Métodos: Trata-se de um estudo de caráter descritivo e de natureza qualitativa, pois considera-se a interpretação dos fatos a partir da vivência cotidiana dos profissionais de saúde. Os dados foram coletados por meio de uma entrevista semiestruturada com enfermeiros dos setores cirúrgicos, e analisados na modalidade análise de conteúdo, diante de suas três fases: pré-análise, exploração do material e a interpretação dos mesmos. Resultados: os resultados obtidos foram classificados em dois núcleos de sentido, sendo eles: importância e benefícios da visita pré-operatória e aspectos gerenciais da visita pré-operatória. Conclusão: a fragmentação do processo de trabalho, a falta de investimentos e de recursos, bem como de uma prática baseada em evidência ainda é uma realidade. Neste sentido, são necessários investimentos para uma maior e melhor articulação multiprofissional para com os aspectos referentes à segurança do paciente cirúrgico.

Palavras-chave: Enfermagem perioperatória, Cuidados pré-operatórios, Papel do profissional de enfermagem.

\section{ABSTRACT}

Objective: to analyze the nurses' perception of the operationalization of the preoperative visit and to identify the understanding and difficulties to carry it out. Methods: This is a descriptive and qualitative study, as the interpretation of facts from the daily experience of health professionals is considered. The data were collected through a semi-structured interview with nurses from the surgical sectors, and analyzed in the content analysis modality, considering its three phases: pre-analysis, exploration of the material and their interpretation. Results: the results obtained were classified into two cores of meaning, namely: importance and benefits of the preoperative visit and managerial aspects of the preoperative visit. Conclusion: the fragmentation of the work process, the lack of investments and resources, as well as an evidence-based practice is still a reality. In this sense, investments are needed for greater and better multiprofessional articulation with regard to aspects related to the safety of surgical patients.

Key words: Perioperative nursing, Preoperative care, Nurse's role.

\section{RESUMEN}

Objetivo: analizar la percepción de las enfermeras sobre la operacionalización de la visita preoperatoria e identificar la comprensión y las dificultades para llevarla a cabo. Métodos: Este es un estudio descriptivo y cualitativo, ya que se considera la interpretación de los hechos de la experiencia diaria de los profesionales de la salud. Los datos fueron recolectados a través de una entrevista semiestructurada con enfermeras de los sectores quirúrgicos, y analizados en la modalidad de análisis de contenido, considerando sus tres fases:

1 Universidade do Oeste Paulista, Presidente Prudente - SP. *E-mail: marceloalexandrealbino@hotmail.com 
preanálisis, exploración del material y su interpretación. Resultados: los resultados obtenidos se clasificaron en dos núcleos de significado, a saber: importancia y beneficios de la visita preoperatoria y aspectos gerenciales de la visita preoperatoria. Conclusión: la fragmentación del proceso de trabajo, la falta de inversiones y recursos, así como una práctica basada en evidencia todavía es una realidad. En este sentido, se necesitan inversiones para una mayor y mejor articulación multiprofesional con respecto a aspectos relacionados con la seguridad de los pacientes quirúrgicos.

Palabras clave: Enfermería perioperatoria, Atención preoperatoria, Rol del profesional de enfermería.

\section{INTRODUÇÃO}

O Centro Cirúrgico é um setor restrito onde são realizadas cirurgias de alta, média e baixa complexidade. As tarefas desenvolvidas neste setor são divididas em três períodos: pré-operatório, intraoperatório e pósoperatório, que juntos são chamados de perioperatório (RIBEIRO E, et al., 2017; CHEEVER KH e HINKLE JL, 2017).

A Sistematização da Assistência de Enfermagem Perioperatória (SAEP) é utilizada como forma de operacionalização do Processo de Enfermagem à pessoa cirúrgica no período perioperatório, que compreende o momento de 24 horas antes da cirurgia até as primeiras 48 horas após o ato anestésicocirúrgico, este instrumento dispõe de cinco etapas: pré-operatório imediato, transoperatório, recuperação pósanestésica, pós-operatório imediato e pós-operatório mediato (OLIVEIRA MM e MENDONÇA KM, 2014).

A admissão da pessoa no setor cirúrgico é função do enfermeiro, que tem como principal objetivo proporcionar conforto e segurança de forma humanizada, com consequente minimização do medo e ansiedade. Tais fatores são ocasionados por diversos fatores, destacando-se o ambiente cirúrgico e o procedimento invasivo (ROCHA IF e PINCERATI CLA, 2017).

A ansiedade é um elemento comum e esperado na experiência cirúrgica; este sentimento desencadeia medo e estresse, sendo o principal motivo para a retenção de informações necessárias e alterações fisiológicas como, por exemplo, o aumento do débito cardíaco. Desta forma, cabe ao enfermeiro realizar intervenções educacionais e de apoio que buscam proporcionar o alívio e conforto ao acolher a pessoa e sua família (BAREL PS, et al., 2018).

Por ser um setor complexo onde ocorrem diversas intervenções emergenciais, os profissionais deste local acabam, na maioria das vezes, sobrecarregados, o que impacta diretamente na qualidade do serviço prestado. Desta forma, a admissão da pessoa no centro cirúrgico acaba não sendo priorizada, o que interfere diretamente no objetivo de mantê-la confortável e preparada para o procedimento (SORATTO MT, et al., 2017).

Soratto MT, et al. (2015), Siqueira NS e Schuh LX (2017) afirmam que a falta de admissão do paciente no centro cirúrgico, bem como a não realização da visita pré-operatória de enfermagem pelo enfermeiro tem como causas a sobrecarga de trabalho, a falta de planejamento do serviço, e o conhecimento superficial sobre a importância da assistência de enfermagem cirúrgica a pessoa e sua família.

O presente estudo tem relevância para a área da saúde, pois pesquisas demonstram que o esclarecimento de dúvidas dos pacientes sobre o momento cirúrgico, bem como o papel da enfermagem para promoção do cuidado e da segurança são essenciais para redução da ansiedade extrema com consequente melhora na recuperação pós-operatória (GONÇALVES TF e MEDEIROS VCC, 2016; GONÇALVES MAR, et al. 2017).

Desta forma, identifica-se que há fragilidades na operacionalização da SAEP nos serviços de saúde, em especial, na realização da visita pré-operatória de enfermagem, sendo esta prática crucial e importante para promoção do cuidado e prevenção de complicações inerentes ao ato cirúrgico, justificando, assim, a necessidade de melhor atendimento a pessoa cirúrgica e sua família. Assim, o objetivo deste estudo é analisar a percepção dos enfermeiros sobre a operacionalização da visita pré-operatória, ao identificar o entendimento e dificuldades para executá-la.

REAS/EJCH | Vol.Sup.n.42 | e2842 | DOI: https://doi.org/10.25248/reas.e2842.2020 Página 2 de 8 


\section{MÉTODOS}

Trata-se de uma pesquisa de campo, de caráter qualitativo-descritivo. As fases da pesquisa foram realizadas de forma a considerar a interpretação dos fatos e fenômenos que ocorrem dentro de seus nichos, cenários e ambientes naturais de vivência. A opção por esta abordagem permite uma compreensão mais ampla, valorizando as opiniões de cada colaborador (AUGUSTO CA, et al., 2013).

O local do estudo foi um hospital do oeste paulista que possui uma megaestrutura com diversos setores, contendo no total 550 leitos e três centros cirúrgicos: no primeiro são realizados atendimentos para procedimentos complexos e de urgência e emergência; no segundo há intervenções de baixa complexidade para cirurgias ambulatoriais; e no terceiro, procedimentos ginecológicos e obstétricos.

A população de estudo foi composta por enfermeiros que exercem sua prática profissional nos setores cirúrgicos e que respeitaram o critério de inclusão de trabalhar, no mínimo, um ano em sua função. A amostra foi composta por 17 enfermeiros, incluindo os assistenciais e gestores dos setores cirúrgicos, que aceitaram participar e assinaram o Termo de Consentimento Livre e Esclarecido (TCLE) em consonância aos princípios éticos da pesquisa.

A coleta de dados foi realizada no próprio hospital pelos autores no período de maio a julho de 2019 por meio de uma entrevista semiestruturada e registrada por meio de gravação com aparelho telefônico. Após, foram transcritas para análise. O tempo médio das entrevistas foi de 15 minutos. Os participantes foram enumerados aleatoriamente, respeitando o sigilo das informações. As perguntas norteadoras da entrevista abordaram a importância que os enfermeiros atribuem para realização da visita pré-operatória, bem como as dificuldades encontradas por eles no dia-a-dia para executá-la.

Os dados foram analisados por meio da análise de conteúdo. A pré-análise foi realizada de forma a organizar os dados obtidos. Após, os mesmos foram explorados, classificados e categorizados em consonância com o objetivo deste estudo, de forma a enunciar os resultados de forma crítica e reflexiva (BARDIN L, 1977). Ressalta-se que os dados só foram coletados após aprovação do Comitê de Ética em Pesquisa (CEP) com número do parecer 3.255.175 e CAE 10180219.4.0000.5515.

\section{RESULTADOS e DISCUSSÃO}

Dos 17 enfermeiros abordados para participarem da pesquisa, um estava de férias, um havia sido promovido para outra função, três não entraram no critério de inclusão, pois não tinham o tempo mínimo de experiência; e um se recusou a participar pois não houve interesse na participação. Desta forma, a amostra final foi composta por 11 enfermeiros. Com a análise dos dados foi possível identificar dois núcleos de sentido: "Importância e benefícios da visita pré-operatória"; e "Aspectos gerenciais da visita pré-operatória".

\section{Importância e benefícios da visita pré-operatória}

Os enfermeiros relataram que a visita pré-operatória é importante para identificar os riscos cirúrgicos do paciente, bem como para o esclarecimento de dúvidas quanto aos cuidados de enfermagem para 0 procedimento cirúrgico.

"[...] e a visita pré-operatória é de grande importância pro paciente porque ela minimiza os riscos que o paciente está exposto durante processo cirúrgico". E2

"[...] então a gente passa visita para esclarecer as dúvidas deles da parte da enfermagem, nossa parte, tipo exame, RX, ECG, que deve ser feito naquele momento a gente já informa". E1

A ansiedade é o principal sentimento que ocorre nos pacientes que passaram por um procedimento anestésico-cirúrgico. Em um estudo realizado na Etiópia, houve prevalência de $47 \%$ de pessoas com ansiedade pré-operatória. Os principais fatores desencadeantes dos níveis estressores são o tempo de espera, o medo da ocorrência de erros relacionados a procedimentos médicos e de enfermagem (BEDASSO A e AYALEW M, 2019). 
Além disso, o apoio e a presença aos familiares neste período são essenciais, pois estes passam segurança e auxiliam nos cuidados prestados à pessoa cirúrgica. Desta forma, cabe a enfermagem ofertar não só um cuidado técnico/cientifico, mas também humano e integral. Contudo os entrevistados não associaram a ansiedade como um fator de risco, ou como um aspecto importante a ser abordado (GONÇALVES TF e MEDEIROS VCC, 2016; COSTA TMN e SAMPAIO CEP, 2015).

Koch TM, et al. (2018), alegam que a visita pré-operatória deve ser uma ferramenta eficaz e potencializadora do cuidado, levando o bem-estar e também oferecendo auxílio no planejamento da assistência contínua e individualizada. Destacam também que sua ausência é o principal motivo do cancelamento da cirurgia, tendo em vista que, um de seus objetivos, é obter informações clínicas para ocorrência do ato anestésico/cirúrgico, aspecto este identificado pelos entrevistados.

Outro aspecto importante identificado pelos enfermeiros foram os cuidados específicos empregados durante a operacionalização da visita pré-operatória:

"[...]Vai fazer qualquer tipo de esclarecimento, tempo de cirurgia, tipo de anestesia isso seria meu entendimento sobre a visita pré-operatória." E1

"[...] Explica pra ele como vai ser o procedimento, e preparo pro procedimento, o jejum, banho, explicar o pós-operatório que ele pode voltar da cirurgia com um dreno por exemplo, acho que isso é um momento que tira mesmo as dúvidas do paciente, e orienta ele." E11

Os cuidados de enfermagem durante a visita pré-operatória são essenciais para promoção de um cuidado individualizado e humanizado. Desta forma, não devem ser limitados a orientações, mas sim em intervenções capazes de promover o alívio das angústias e promoção da segurança da pessoa cirúrgica (KOSH TM, et al., 2018). No ano de 2010, em consonância com a aliança mundial para a segurança do paciente, a Agência Nacional de Vigilância Sanitária (ANVISA) publicou o manual Cirurgias Seguras Salvam Vidas contendo os dez objetivos essenciais para a cirurgia segura (BRASIL, 2009).

Dentre os objetivos, no que diz respeito ao período pré-operatório, destaca-se a atuação da equipe cirúrgica ao identificar de forma segura o paciente, operando-o no local cirúrgico certo. Outro objetivo são os métodos sistemáticos para minimizar o risco de infeção do sítio cirúrgico e o controle da dor; tais medidas precisam ser iniciadas e orientadas já na visita pré-operatória de enfermagem, a fim de preparar o paciente para 0 ato anestésico-cirúrgico. Desta forma, é de suma importância que a visita pré-operatória de enfermagem permita o cumprimento destes objetivos, a fim de que se promova a segurança do paciente cirúrgico (BRASIL, 2009).

Realizar esclarecimentos de forma a acolher e elucidar as dúvidas é essencial para uma prática de enfermagem humanizada; além disso, é crucial que esta prática seja baseada em evidências, uma vez que, a enfermagem é uma ciência. Desta forma, o encontro do enfermeiro com a pessoa cirúrgica não deve se limitar a um checklist de segurança cirúrgica (CSC), mas sim a um ato de cuidado e atenção em saúde (KOSH TM, et al., 2018).

Costa TMN e Sampaio CEP (2015), bem como Otaviano MLP, et al., (2016) elucidam que as intervenções realizadas pela enfermagem no período pré-operatório causam conforto ao paciente, criam vínculo, reduzem o tempo de internação, as infecções hospitalares e as complicações pós-operatórias. Desta forma, é fundamental que a visita pré-operatória de enfermagem ocorra de forma ampliada e devidamente fundamentada. Além das intervenções apontadas pelos enfermeiros é importante incluir cuidados de enfermagem referente ao uso de adornos, próteses, esvaziamento vesical, higiene corporal, uso de camisola e gorro hospitalar e a realização de tricotomia conforme procedimento operacional da instituição; todos estes aspectos não foram mencionados pelos enfermeiros.

Para que as intervenções de enfermagem tenham impacto, exige-se do enfermeiro habilidades de comunicação, pois as informações devem ser passadas de forma clara e de fácil compreensão. Uma pesquisa realizada por Rocha DR e Ivo OP (2015) mostra que $67 \%$ dos pacientes entrevistados não compreendiam as 
orientações passadas pela enfermagem. Dentre as causas, os autores apontam a escolaridade inferior e os aspectos culturais; entretanto é importante o uso de técnicas e habilidades de comunicação, de forma a tornar o encontro da pessoa cirúrgica com a enfermagem, uma oportunidade para promoção da saúde e prevenção de complicações.

Nesta lógica, os entrevistados associaram os cuidados de enfermagem prestados no período préoperatório com os cuidados no período pós-operatório:

"Todo o processo do pós-operatório vai ser mais eficaz, quando ele sabe os cuidados que ele deve ter com a própria saúde[...]." E6

"Em relação ao pós-operatório, eu acho assim, toda relação, se a gente conhece o paciente na entrada a gente vai sabe como dá a conduta depois do centro cirúrgico[...]." E7.

"O pós-operatório vai ajudar a gente vê como o paciente estava antes da cirurgia e preparar os cuidados, para que ele possa se recuperar depois." E9

No pós-operatório o paciente pode desenvolver complicações de origem respiratória, circulatória e gastrointestinal, sendo crucial seu monitoramento, em especial, nas primeiras horas do ato anestésico/cirúrgico. Deve-se realizar o controle de glicemia, normotermia e exercícios respiratórios, além de todos os cuidados aos quais o paciente não consegue executar devido ao ato cirúrgico, como os de higiene corporal, curativos, nutricionais e medicamentosos. Otaviano MLP, et al., (2016) e Kosh TM, et al., (2018) afirmam que um período pré-operatório fragilizado está totalmente vinculado a complicações pós-operatórias.

Desta forma, a importância e os benefícios da visita pré-operatória de enfermagem são inquestionáveis, uma vez que, além de promover o conforto da pessoa cirúrgica, a capacitam para passar pelo ato anestésico/cirúrgico de forma segura, humanizada e com minimização de riscos.

\section{Aspectos gerenciais da visita pré-operatória}

Dada a importância e os benefícios da visita pré-operatória de enfermagem, os enfermeiros relatam aspectos do processo de trabalho que dificultam e limitam sua operacionalização, como, por exemplo, a sobrecarga de trabalho e a falta de enfermeiros:

"A gente não tem um tempo muito longo para poder conversar com o paciente, tira todas as dúvidas, e seria em relação ao tempo mesmo" (E9)

"Se dois enfermeiros, um só pra essa parte mais assistencial mesmo, e outro fica mais na parte burocrática, eu sei que isso infelizmente não é possível né, a gente tenta fazer o melhor." (E4)

"A questão mais seria a instituição né, porque falta funcionário, se fosse assim, dois enfermeiros de dia." (E3)

Os aspectos mencionados pelos enfermeiros citam a falta de recursos humanos e tempo para realização da visita pré-operatória. É importante salientar que a visita pré-operatória faz parte da SAEP, tida como estratégia de operacionalização do Processo de Enfermagem. Desta forma, quando não executada, compromete o cuidado em saúde, expondo os pacientes a situações potencialmente evitadas.

Neste sentido, em revisão integrativa, Oliveira MM e Mendonça KM (2014) identificam estudos que utilizam checklists operatórios como ferramenta para organização do processo de trabalho no que diz respeito ao paciente cirúrgico. Não há um consenso dos aspectos que devem compor estes checklists, uma vez que, estes dependem do perfil epidemiológico, das estratégias utilizadas e prioridades do corpo gestor das instituições de saúde.

Da mesma forma, Andrade JS, et al., (2017) construíram e validaram um instrumento a partir da teoria das necessidades humanas básicas de Wanda Horta, contendo os aspectos psicobiológicos, psicossociais e psicoespirituais que envolvem o paciente cirúrgico. Monteiro ED, et al., (2014) construíram um instrumento 
contendo informações de todos os períodos operatórios, ressaltando que cabe ao enfermeiro do setor de internação enunciar os diagnósticos de enfermagem, pois entenderam que é na clínica de internação onde há maior tempo hábil para a coleta de dados dos pacientes.

Alpendre FT, et al., (2017) elaboraram um instrumento que contempla os períodos pré e pós-operatório, avançando para o quesito complicações e alta hospitalar. Mazzi e Tonhom (2014) argumentam a necessidade de instrumentos de caráter mais qualitativo, de forma que o registro das informações contemple, de fato, os cuidados específicos da enfermagem e não todo o processo de avaliação.

No local de estudo desta pesquisa, o instrumento usado no período transoperatório é denominado de SAEP e não contempla os períodos pré e pós-operatórios. Há um procedimento operacional padrão que institui um checklist pré-operatório contendo cuidados importantes que devem ser implementados no período pré-operatório, porém os enfermeiros relatam desconhecê-lo:

"Então se realmente estiver o protocolo é uma falta de interesse nosso de olhar mais se tiver o protocolo vai ajudar" E3

Nota-se que os instrumentos utilizados ainda não conseguem dimensionar a complexidade dos fenômenos de enfermagem do paciente cirúrgico, bem como de fato não estão instituídos como estratégias de organização do processo de trabalho.

O uso das teorias de enfermagem como referencial para o cuidado do paciente cirúrgico é um caminho assertivo, visto que estas descrevem, preveem e identificam padrões para a prática de enfermagem. Sem a definição de uma teoria de enfermagem que norteie a coleta dos dados, definição do diagnóstico de enfermagem e intervenções, os instrumentos produzidos e a própria prática de enfermagem em si tornam-se superficiais, desordenadas e fragmentadas (MCEWEN M e WILLS EM, 2016). Além disso, o cuidado ao paciente cirúrgico ainda ocorre de forma não articulada com as demais profissões, como exposto abaixo:

"A comunicação entre as equipes multidisciplinares e entre outros e serviços de apoio também, laboratório, banco de sangue precisa ocorrer" E2

O cuidado multiprofissional é uma realidade já experienciada em muitas instituições de saúde. No que diz respeito ao paciente cirúrgico, a equipe anestésica também realiza a avaliação pré-anestésica, conforme aponta a Resolução do Conselho Federal de Medicina (CFM) № 1802, de 4 de outubro de 2006. Além dos anestesiologistas, é importante que as demais profissões da saúde estejam presentes no período préoperatório, a fim de, também, programarem seus respectivos cuidados.

É comum também a articulação dos instrumentos de coleta de dados cirúrgicos estarem vinculados com o checklist de verificação de cirurgias seguras proposto pela Organização Mundial de Saúde (OMS) em 2004; isto após o lançamento da campanha chamada "Cirurgias Seguras Salvam Vidas" (OMS, 2009). Articular os cuidados de enfermagem no perioperatório com o checklist de verificação de cirurgia segura e com os cuidados multiprofissionais ainda é um grande desafio. $O$ uso de instrumentos e checklists é uma tendência nos serviços de saúde, porém, é importante ressaltar que seu uso não garante a qualidade dos cuidados prestados, e sim, somente a organização do processo de trabalho quando devidamente implementado.

Desta forma, é de suma importância que os gestores, comprometidos com a qualidade dos serviços de saúde e com a segurança do paciente, apropriem-se de seu perfil epidemiológico e dos aspectos culturais que estão presentes em suas organizações, de forma a considerá-los ao organizarem o processo de trabalho de seus profissionais.

A tomada de decisões deve ser coletiva e partilhada com os profissionais, de forma a envolvê-los no processo, pois são eles quem executarão a melhor forma de implementação dos cuidados aos pacientes cirúrgicos (GOMES CDPP, et al, 2016). Por fim, o investimento em educação em saúde e educação permanente devem ser tidos como estratégias gerenciais essenciais para garantia da missão e valores das organizações de saúde, de forma que a qualidade assistencial esteja sempre presente (MARTINS FZ, DALL"AGNOL, 2016). 


\section{CONCLUSÃO}

A importância e os benefícios da visita pré-operatória de enfermagem são inquestionáveis. Infelizmente, a fragmentação do processo de trabalho, a falta de investimentos e de recursos, bem como de uma prática baseada em evidência ainda é uma realidade. Neste sentido, a articulação multiprofissional com os aspectos referentes à segurança do paciente cirúrgico ainda é um grande desafio. Cabem às organizações de saúde definirem suas prioridades e definirem que tipo de cuidado querem ofertar; ressalta-se aqui que a organização do processo de trabalho da enfermagem devidamente fundamentada é uma potente estratégia, visto que a enfermagem é a profissão mais articulada e capacitada para este fim.

\section{REFERÊNCIAS}

1. ALPENDRE FT, et al. Cirurgia segura: validação de checklist pré e pós-operatório. Rev. Latino Americano de Enfermagem. Ribeirão Preto, 2017; 25.

2. ANDRADE JS, et al. Sistematização da assistência de enfermagem perioperatória: validação de um instrumento de coleta dados. Prática assistencial de enfermagem. Portal COFEN, 2017.

3. AUGUSTO CA, et al. Pesquisa qualitativa: rigor metodológico no tratamento da teoria dos custos de transação em artigos apresentados nos congressos da sober (2007-2011). Revista de economia e sociologia rural. Brasília, 2013; 51(4).

4. BARDIN L. Análise de conteúdo. Lisboa: Edições 70, 1977.

5. BAREL PS, et al. Anxiety and knowledge of patients before being subjected to orthognathic surgery. Brasília: Rev. Brasileira Enfermagem, 2018; 71.

6. BEDASSO A, AYALEW M. Preoperative anxiety among adult patients undergoing elective surgery: a prospective survey at a general hospital in Ethiopia. Rev. Paciente Saf Surg, 2019; 18.

7. BRASIL. Ministério da Saúde. Segundo desafio global para a segurança do paciente: cirurgias seguras salvam vidas (orientações para cirurgia segura da OMS) / Organização Mundial da Saúde - Rio de Janeiro: Organização Panamericana de Saúde, 2009.

8. CHEEVER KH, HINKLE JL. Brunner e Suddarth: Tratado de enfermagem médico-cirúrgica. 13 ed. Rio de janeiro: Guanabara Koogan, 2017.

9. COSTA TMN, SAMPAIO CEP. As orientações de enfermagem e sua influência nos níveis de ansiedade dos pacientes cirúrgicos hospitalares. Revista Enfermagem, Rio de Janeiro, 2015; 23(2).

10. CREMESP. 2006. Conselho Federal de Medicina. Resolução CFM no 1.802, 4 de outubro de 2006.

11. GOMES CDPP, et al. Percepção de uma equipe de enfermagem sobre a utilização do checklist cirúrgico. Rev. Sobecc. São Paulo, 2016, 21(3): 140-145

12. GONÇALVES MAR, et al. A influência da informação fornecida pelos enfermeiros sobre a ansiedade pré-operatória. Rev. Enf. Ref, Coimbra, 2017, 14.

13. GONÇALVES TF, MEDEIROS VCC.A visita pré-operatória como fator atenuante da ansiedade em pacientes cirúrgicos. Rev. Sobecc, 2016; 21(1):22-27.

14. KOSH TM, et al. Momento Anestésico-Cirúrgico: Transitando entre o conhecimento dos(as) enfermeiros(as) e o cuidados de enfermagem. Rev. Sobecc, São Paulo, 2018; 23(1):7-13.

15. MARTINS FZ, DALL'AGNOL CM. Centro cirúrgico: desafios e estratégias do enfermeiro nas atividades gerenciais. Rev. Gaúcha de Enfermagem. 2016. 37(4)

16. MAZZI NR, TONHOM SFR. Visita pré-operatória de enfermagem: validação de um instrumento. Revista Electronica Acervo Saúde, 2014, 6(2):638-645.

17. MCEWEN M, WILLS E. Bases teóricas da Enfermagem. 4 ed. Porto Alegre: Artmed, 2016, 590 p.

18. MONTEIRO ED, et al. Cirurgias seguras: elaboração de um instrumento de enfermagem perioperatória. Rev. Sobecc, São Paulo, 2014, 19(2).

19. OLIVEIRA MM, MENDONÇA KM. Análise da visita pré-operatória de enfermagem: revisão integrativa. Rev. Sobecc, São Paulo, 2014, 19(2).

20. ORGANIZAÇÃO MUNDIAL DA SAÚDE. Segundo desafio global para a segurança do paciente: Cirurgias seguras salvam vidas (orientações para cirurgia segura da OMS). Rio de Janeiro: Organização Pan-Americana da Saúde; Ministério da Saúde; Agência Nacional de Vigilância Sanitária, 2009.

21. OTAVIANO MLP, et al. Prevenção de infecção do sítio cirúrgico. Rev. Aletheia, 2016, 49(2):144-146.

22. RIBEIRO E, et al. Atitudes dos enfermeiros de centro cirúrgico diante a sistematização de enfermagem perioperatória. Rev. Sobecc, São Paulo, 2017, 22(4). 
23. ROCHA DR, IVO OP. Assistência de enfermagem no pré-operatório e sua influência no pós-operatório. Uma percepção do cliente. Revista Enfermagem Contemporânea, 2015, 4(2):170-178.

24. ROCHA IF, PINCERATI CLA. O papel do enfermeiro na admissão do paciente no centro cirúrgico. Relatório final Fundação Educacional do Município de Assis, Assis, 2017.

25. SIQUEIRA NS, SCHUH LX. As atribuições do enfermeiro no centro cirúrgico. Universidade Luterana do Brasil Cachoeira do Sul, 2017.

26. SORATTO MT, et al. O estresse da equipe de enfermagem no centro cirúrgico, 2016, 5(1):179-192. 